\title{
VIEJAS PREGUNTAS, ¿VIEJAS RESPUESTAS?: ALGUNAS REFLEXIONES EN TORNO A LA CONVERGENCIA
}

\author{
Luis Bértola*
}

\section{INTRODUCCIÓN}

Se me ha invitado a hacer una presentación de veinte minutos sobre temas de historia económica internacional. Pretendo presentar unas pocas ideas en torno a las estrategias para abordar el problema del desigual desempeño económico de las naciones y presentar alguna evidencia empírica con el fin de ilustrar una posible estrategia de investigación.

El debate en torno a la convergencia de tasas de crecimiento y de los precios de diferentes factores de producción no es un recién llegado. La literatura al respecto es ya abundante, al igual que la cantidad de evidencia empírica manejada. En primer lugar, entonces, presentaremos escuetamente el debate (sección 1).

Desarrollaremos nuestro argumento sobre el trasfondo del desarrollo de Argentina, Brasil y Uruguay en relación a los países desarrollados en 1870-1998. Para ello presentaremos, en segundo lugar, algunos indicadores de desarrollo relativo (sección 2).

Un tema central de la discusión en torno al desempeño de nuestros países ha sido la 'pertinencia y consecuencias de las políticas de diversificación productiva. Sin entrar a este tema de manera directa, buscaremos ejemplificar nuestra estrategia de investigación e hipótesis de trabajo con la discusión del éxito relativo de las economías del Río de la Plata

* Programa de Historia Económica y Social, Facultad de Ciencias Sociales, Universidad de la República-Uruguay. Este artículo es fruto de un ya largo proceso de trabajo y reflexión junto a Gabriel Porcile; es, en realidad, una coautoría. A él se deben muchos de los méritos de este trabajo. Los errores y carencias me corresponden por entero.lbertola@fcsum.edu.uy 
antes de 1930, con evidencia principalmente del caso uruguayo, para el que disponemos de mayor información (sección 3).

\section{EL DEBATE Y LAS ESTRATEGIAS SEGUIDAS: $;$ MUCHO RUIDO Y POCAS NUECES?}

En un interesante trabajo de síntesis teórica y empírica sobre el estado de la teoría del crecimiento y del debate en torno a la convergencia, Angel de la Fuente concluye que en sentido estricto ningún modelo de crecimiento, ya sea con cambio tecnológico endógeno o exógeno, ha logrado capturar y explicar la dinámica del crecimiento relativo de las naciones y las regiones, por lo que la teoría del crecimiento y, más aún la teoría del desarrollo, muestra todavía enormes lagunas (DE LA FUENTE, 1996, II, p. 100-105).

Forzando las cosas, como es menester realizar en "zipeos" del estado de la cuestión como el que aquí hacemos, las grandes estrategias, desde un punto de vista metodológico, parecen haber seguindo al menos dos grandes caminos.

Una estrategia ha partido de esbozar modelos de crecimiento y ha buscado luego contrastarlos con la evidencia empírica. La aparición de "anomalías" empíricas ha generado un proceso continuo de corrección de los modelos para limpiar los hechos de discrepancias con respecto a los supuestos originales del modelo. Este el caso, principal pero no exclusivamente, de la teoría neoclásica y sus predicciones de convergencia internacional de tasas de crecimiento y precios de factores por efecto de los rendimientos decrecientes. Los modelos neoclásicos del crecimiento fueron progresivamente corregidos para explicar el residual, introduciendo, entre cosas, diferentes especificaciones del capital: capital humano, esfuerzos en R\&D, etc. En lo que respecta específicamente al debate sobre convergencia, la estrategia ha sido la de ir corrigiendo desviaciones de los supuestos básicos: por ejemplo, aspectos institucionales y tasas de inversión dentro de una gama creciente de aspectos. Williamson ha optado por señalar que la discusión sobre convergencia no debe darse sobre la base del "artefacto estadístico" llamado PBI, sino a partir de precios de factores como el salario (WILLIAMSON, 1995). Cuando al medir la convergencia de salarios se encuentran las esperadas anomalías, el modelo busca limpiar los hechos: corrección por efectos demográficos y de distribución del ingreso vinculados a las grandes migraciones, fallas institucionales, etc. (WILLIAMSON, 1998). En síntesis, la estrategia es depurar los hechos para probar la validez de la 
teoría. Al decir de un colega, si dejamos la historia a un lado, la convergencia existe. La estrategia de investigación no está centrada en recurrir a la teoría para explicar el desarrollo histórico sino en construir un escenario empírico virtual en el cual la teoría se vea ratificada.

Sin caer en el historicismo, la narrativa, el apego incondicional a los hechos y un enfoque autárquico y/o particularista del desarrollo económico, otras corrientes del pensamiento económico han intentado partir de hechos estilizados y recurrir a la teoría económica para explicarlos. La propia jeraquización de los hechos a explicar forma parte de la construcción teórica y la teoría, por tanto, adquiere un carácter historizado. Sin dudas existen nexos importantes entre las opciones metodológicas y las teorías manejadas. Los hechos estilizados que se pretenden explicar guardan relación con las opciones teóricas. No cabe dudas que la jerarquización del estudio de los procesos de cambio tecnológico ha provenido de vertientes heterodoxas del pensamiento económico, al igual que el estudio de los aspectos institucionales. En lo que refiere más concretamente al campo de los procesos de convergencia y divergencia, este tipo de orientación tiene dificultades para encontrar explicaciones sencillas y leyes generales. El conjunto de variables movilizadas para interpretar los procesos se incrementan y la complejidad de las mismas dificultan el modelaje. Tal vez pueda decirse que en esta dirección de trabajo debe aspirarse más a estar "vagamente acertados antes que precisamente equivocados", por citar una elocuente expresión de Crafts (1999). Pero ello no es excusa para una vaga formulación teórica.

Nuestro trabajo aspira a inscribirse en esta segunda línea de investigación. Algunas referencias teóricas básicas son las siguientes:

- Se pone el énfasis del desarrollo económico en los procesos de innovación y en el ingenio del hombre y sus efectos sobre el crecimiento de la productividad antes que en la asignación de recursos escasos (PASINETTI, 1983).

- El cambio tecnológico es un proceso desparejo, con fuertes determinantes institucionales, que genera respuestas subóptimas por parte de agentes que actúan con racionalidad limitada y que inician trayectorias tecnológicas de difícil previsión. Las ventajas comparativas de las naciones son resultado de los procesos de construcción institucional, de los recorridos que adoptan los procesos de innovación y de las características sistémicas de dichos procesos (NELSON, 1992; NELSON, 1994; LUNDVALL, 1992). 
- El crecimiento económico conlleva un proceso de cambio estructural, determinado por factores tanto de oferta como de demanda. En tanto el cambio tecnológico avanza de manera diferente en distintos sectores y se difunde a diferentes ritmos, la productividad aumenta de manera igualmente despareja y los movimientos de precios relativos readecuan las relaciones sectoriales. Igualmente, los movimientos de los precios relativos y los cambios en los niveles de ingreso promueven cambios en la estructura de la demanda que interactúan con los de la estructura productiva. (PASINETTI, 1980).

- Los frutos del progreso técnico no se distribuyen exclusivamente de manera clásica, es decir, por vía de la reducción de los precios. Un estímulo de la innovación debe ser, mediando derechos de propiedad claramente establecidos (North), la posibilidad de apropiar los frutos de la innovación de manera colusiva (Reinert). Por lo tanto, la tecnología no es de libre acceso ni los frutos del progreso técnico se permean a través de los precios. El grado y la forma en que lo harán estará determinado por las particularidades de la tecnología vigente, de las estructuras de mercado y de los diferentes arreglos institucionales vigentes.

- En una economía internacional se reproducen las desigualdades del desarrollo tecnológico y de los niveles de productividad vigentes en cada rama industrial. La velocidad con que se difunde el cambio tecnológico dependerá de la magnitud de la brecha y de las aptitudes para apropiarse de la nueva tecnología. La brecha tecnológica es una fuente de crecimiento relativo, pero la capacidad de apropiarla estará igualmente determinada por los esfuerzos domésticos de aprendizaje (Verspagen).

- Los elementos anteriormente expuestos pueden ser articulados en torno a una propuesta básica, de inspiración kaldoriana tanto como cepalina, formalizada por Thirlwall (1983) y reinterpretada por Bértola y Porcile (1998).

Sobre la base de un modelo simplificado de crecimiento económico con equilibrio de la balanza de pagos, podemos establecer una propuesta 
básica acerca de las condiciones para el desempeño relativo de un país con respecto al resto del mundo de acuerdo a la siguiente ecuación:

$$
\frac{\mathrm{yi}_{\mathrm{i}}^{*}}{\mathrm{z}}=\frac{\varepsilon_{\mathrm{i}}}{\pi_{\mathrm{i}}}
$$

donde yi* es la tasa de crecimiento del ingreso con equilibrio de la balanza de pagos del país "i" (abstrayendo de los movimientos de precios y de los flujos de capital), z es la tasa de crecimiento en el resto del mundo, ci es la elasticidad ingreso de la demanda de las exportaciones de " $\mathrm{i}$ " y $\pi \mathrm{i}$ es la elasticidad ingreso de la demanda de las importaciones de "i". ${ }^{1}$ De acuerdo a la formulación de Bértola y Porcile (1998), estas elasticidades deben ser entendidas como expresión de tres componentes diferentes: el comportamiento de la demanda propiamente dicha a nivel de producto y la competitividad en esos mercados; el nivel de especialización que determina la capacidad de competencia en nuevos mercados y de cambio estructural; y el conjunto de arreglos institucionales que regulan la competencia internacional.

Sobre la base de esta propuesta básica pueden articularse diferentes escenarios de desempeño relativo internacional, que presentaremos después de revisar nuestros hechos estilizados.

\section{LOS HECHOS ESTILIZADOS}

Se presentan a continuación indicadores de desempeño relativo para Argentina, Brasil y Uruguay en relación a cuatro países desarrollados (Alemania, EUA, Francia y Gran Bretaña) en el período 1870-1990.

Los indicadores que utilizaremos serán siempre presentados en términos relativos y son el PBI per capita, el poder de compra de los salarios reales y la cobertura educativa.

El gráfico 1 muestra para cada uno de los tres países del Sur el desarrollo de los tres indicadores referidos. Cabe destacar que el nivel 100 representa el promedio de los cuatro países desarrollados.

1 MCCOMBIE, J. S.; THIRLWALL, A. P. Economic growth and the balance of payments constraint. [S. 1.: s. n., 19-]. Chap. 3 
Los principales hechos que se desprenden de los datos presentados son los siguientes:

\section{Con respecto al PBI per capita}

Argentina y Uruguay muestran niveles relativos altos hasta aproximadamente 1913. A partir de entonces Argentina comienza un prolongado y permanente proceso de deterioro. Este país había partido de niveles originariamente más bajos y logró superar a los países europeos durante las tres primeras décadas del siglo. Con respecto a EUA nunca pudo acortar distancias. Uruguay muestra una permanente tendencia al retraso, que sin embargo encontró algunos paréntesis. Argentina y Uruguay se diferencian nítidamente de Brasil: hasta 1913 porque se distancian de este país; desde entonces Brasil acorta distancias hasta llegar a fines del siglo actual con similares niveles de ingreso per capita. Brasil logra acortar distancias con los líderes desde 1900, de forma muy gradual y conseguir salvar el hiato de manera significativa. En términos relativos a la región su desempeño es destacableable. Un hecho sumamente importante es el liderazgo tomado por EUA que obtiene, mantiene y amplía la brecha de ingresos per capita con respecto al resto de los países por períodos muy prolongados. (Por más discusión ver Bértola y Porcile, 1998).

\section{Con respecto a los salarios reales}

Las estimaciones se basan en mojones históricos para los que se realizaron comparaciones internacionales de capacidad de poder de compra de los salarios en términos de una canasta de alimentos y vivienda similar. Esos mojones, que arrojan posiciones relativas internacionales de los salarios, son luego proyectados por las series nacionales de salarios reales

Los salarios reales internacionales muestran un desarrollo que, en términos generales, coincide con el del PBI per capita. En otras palabras, no encontramos evidencia de que los salarios reales cuenten una historia diferente a la del PBI per capita. A pesar de provenir de fuentes claramente diferentes, los casos uruguayo y argentino muestran muy nítidamente cómo ambas variables experimentan un proceso muy similar. Los gráficos muestran algunos quiebres importantes en las series salariales que se deben a cambios en el mojón de comparación internacional de 1913 a 1975. Al igual que en el caso del PBI per capita, si eliminamos a EUA en el período anterior a la segunda posguerra vemos que los niveles salariales argentinos y uruguayos 
compiten bien con los europeos. Sin embargo no se encuentra una bonificación para que las poblaciones de estos países se muevan al Sur de América, lo que sí encuentran los habitantes de los países del mediterráneo, con respecto a los cuales tienen ventajas muy marcadas (BÉRTOLA; CAMOU; PORCILE, 1999). El caso brasileño muestra un proceso muy prolongado de divergencia entre salarios y PBI per capita hasta la Primer Guerra Mundial. Ello puede resultar de errores en las estimaciones (la base estadística es muy deficiente), o bien, lo que es muy probable, de un proceso de creciente divergencia regional en Brasil: los salarios reales representan a los núcleos de desarrollo (en este caso la economía urbana de Rio de Janeiro), en tanto el PBI expresa al conjunto de una economía que vio desarrollarse de manera espectacular a algunas regiones, en tanto otras muy extensas permanecían con una baja dinámica económica o bien experimentaban procesos regresivos. Igualmente esta divergencia puede expresar un proceso de ajuste de los salarios después de las distorsiones creadas por la abolición de la esclavitud. Otro período en el que los salarios brasileños parecen haber divergido del PBI per capita fue el período del milagro, en el que los salarios en los sectores dinámicos industriales experimentó una importante mejora.

Un hecho llamativo del desarrollo de los países del Río de la Plata hasta aproximadamente 1930 es que los salarios y el PBI per capita muestren desarrollos similares a pesar de que se experimentó un proceso muy marcado de cambios en la distribución del ingreso a favor de las clases terratenientes. Williamson (1998) muestra para el caso argentino (Bértola, Calicchio, Camou y Porcile (1999) lo ratifican e ilustran igualmente para el caso uruguayo) que los precios de la hectárea de tierra desde 1870 hasta algún punto entre 1913 y 1930 crece mucho más rápido que el salario, dando lugar a una distribución regresiva del ingreso. Esto debería llevar a que los salarios disminuyeran su participación en el PBI y, por lo tanto, crecieran a un ritmo menor. La posible explicación de esta contradicción sería que si bien el precio de la tierra aumenta en relación al salario, la masa salarial total aumenta mucho más rápidamente por el empuje de los flujos inmigratorios que la incorporación de nuevos territorios. De todas formas, un estudio más detallado puede profundizar en los procesos de más corto plazo en los que ambas son variables (PBI per capita y salarios no coincidieron).

\section{Con respecto a la cobertura educativa}

Se trata de una variable que presenta diversos problemas de estimación cualitativa, por cierto no mucho mayores que los salarios internacionales. El índice que se presenta en estos gráficos resulta de ponderar 
los estudiantes de enseñanza primaria, secundaria y terciaria por $1,1.4$ y 2 respectivamente, expresando el total en relación al total de la población. Obviamente, el índice sobrevalorará la cobertura en los países con población más joven.

Tres elementos son dignos de destaque en lo referente a la cobertura educativa relativa.

1. Aún cuando también en este aspecto Argentina y Uruguay tienen un punto de partida mucho más favorable que Brasil, llama la atención el marcado retraso de los dos países del Río de la Plata en este plano con respecto a los países líderes, retraso mucho más marcado que en el plano de los salarios y el PBI per capita. Al menos en el caso uruguayo, estas cifras constituyen un desafío fuerte a una creencia muy difundida acerca de las virtudes del sistema educativo.

2. En el período 1920-1970, aproximadamente, se produce un extraordinario esfuerzo educativo en los tres países, que lleva a acortar distancias con los líderes. Más destacable es el caso brasileño. La causalidad entre educación y crecimiento es de difícil tratamiento. Sin embargo, parece ser indiscutible la contribución que realizó el aumento de la cobertura educativa en Brasil para el proceso de acorte de distancias referido anteriormente.

3. A partir de los años ' 60 en Argentina y de los '70 en Uruguay, de manera mucho más marcada en el primero que en el segundo, el deterioro del índice de cobertura educativa acompaña al deterioro de las otras dos variables.

Veamos cómo interpretar estas tendencias convergentes y divergentes. En este trabajo, como se ha adelantado, nos concentraremos en torno al período anterior a 1930. 
GRÁFICO 1 - PBI PER CAPITA (PBI/PC), PODER DE COMPRA DE LOS SALARIOS REALES (SR) Y NIVELES DE COBERTURA EDUCATIVA (CE) DE ARGENTINA, BRASIL Y URUGUAY (PROMEDIO ANUAL DE ALEMANIA, EUA, FRANCIA Y GB=100)
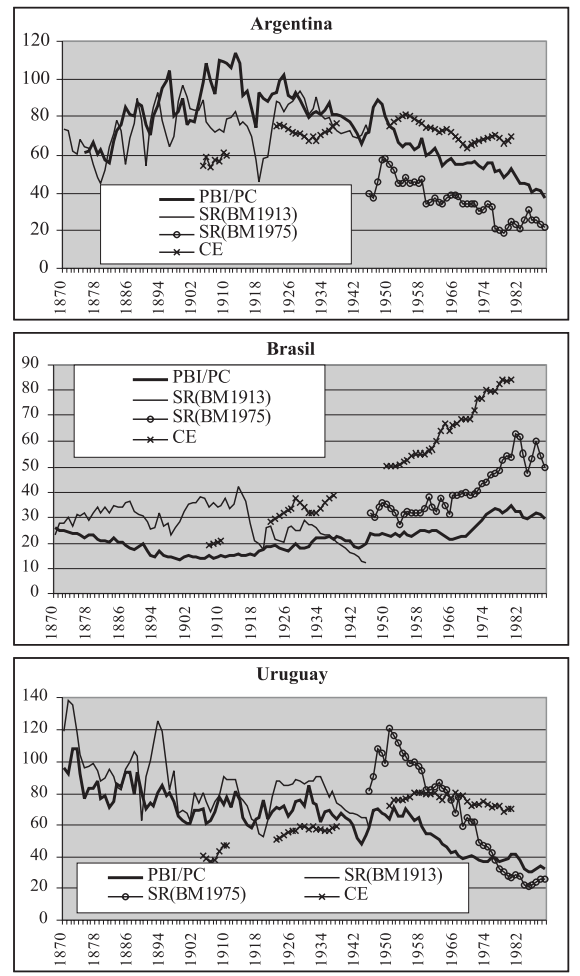

FUENTES: PBI per capita: Bértola y Porcile (1998); salarios reales: Bértola et al. (1999) y Bértola, Camou y Porcile (1999); cobertura educativa: Bértola y Bertoni (1999).

\section{UNA ESTRATEGIA Y ALGUNOS RESULTADOS}

\section{Regímenes de convergencia y divergencia}

Nuestra estrategia de trabajo ha sido la de partir por identificar diferentes escenarios históricos de convergencia y divergencia e intentar 
dar explicaciones adecuadas de los mismos. En Bértola y Porcile, realizamos un primer intento en ese sentido y presentamos una breve tipología de situaciones a partir de manejar un limitado conjunto de variables: tipo de especialización productiva, nivel de apertura económica, régimen de inserción internacional y arreglos domésticos para promover la absorción tecnológica.

De acuerdo a lo que sosteníamos inicialmente, las posibilidades de un país en relación a otros dependerá de la productividad que desarrolle y de las posibilidades de retener los resultados del aumento de ese aumento de la productividad.

Hemos elegido ejemplificar nuestra estrategia con el período 18701930, porque allí se concentra una importante discusión en torno a las determinantes del posterior proceso de divergencia de las economías del Río de la Plata. Podemos sostener que se confrontan, a grandes rasgos, dos posiciones: la de quienes entienden que la divergencia posterior es el resultado de haberse apartado del camino de inserción liberal en la economía mundial, explotando las ventajas comparativas determinadas por la dotación de factores, y la de quienes sostienen que dicho modelo o bien no permitió un desarrollo adecuado o bien estaba agotado, dada la creciente existencia de restricciones externas al crecimiento económico, lo que forzó a la adopción de diversas medidas que aliviaran la restricción externa y promovieran el cambio estructural.

Nuestra posición se encuentra entre estas últimas. Hemos constatado la existencia de escenarios convergentes y divergentes en diversos entornos de apertura y presentado la siguiente enumeración de posibilidades de convergencia:

a) Convergencia con apertura creciente: $\varepsilon>\pi ; \pi>1$;

b) Convergencia con cerramiento: $\varepsilon>\pi ; \pi<1$;

c) Convergencia y estables coeficientes de comercio exterior: $\varepsilon>$ $\pi ; \pi=1 .^{2}$

Recientemente hemos encontrado nueva evidencia de las posibilidades del desarrollo introvertido en el caso brasileño, para el que encontramos que las posibilidades de levantar las restricciones a las importaciones resultaba fundamental para el proceso de crecimiento relativo (BÉRTOLA; BITTENCOURT; PORCILE, 1999).

Veamos algunas hipótesis interpretativas del desempeño anterior a 1930.

2 BÉRTOLA, L.; PORCILE, G. Argentina, Brazil, Uruguay and the world economy: an approach to different convergence and divergence regimes. Montevideo: DT 42/FCS, 1998. p. 6. 


\section{Productividad, precios y salarios en 1870-1930}

La productividad de la producción primario-exportadora de los países de América debe ser abordada en términos de complejos productivos y comerciales que atraviesan diferentes etapas antes de llegar al mercado de consumo.

Podemos desagregar el movimiento de la productividad en cuatro aspectos principales: a) productividad del transporte inter-oceánico, b) la productividad del transporte terrestre, c) la productividad de la propia producción agropecuaria, y d) la reducción de los costos de transacción por concepto de desarrollo y consolidación de aspectos institucionales, particularmente, estabilidad política y vigencia de los derechos de propiedad.

La productividad del transporte inter-oceánico ha sido el principal aspecto considerado por Williamson como determinante de la creación de comercio y en el único en que incorpora sistemáticamente el aumento de la productividad. La reducción de los costos del transporte fue muy importante. Los precios de la tonelada de Buenos Aires a Cardiff cayó un $33 \%$ entre 1870 y $1913 .{ }^{3}$ Dicha caída había comenzado ya en la década de 1840. En términos comparativos con el movimiento general de precios la caída es mucho mayor: Harley (1988) la estima en un 70\% entre 1840 y 1910. Como consecuencia de ello, el "spread" entre los precios en ambas márgenes del Atlántico disminuyó de manera significativa. Según cita Williamson (1998, p. 10), el precio del trigo en Liverpool excedió el de Chicago en un 58\% en 1870 , en $18 \%$ en 1895 y solamente en un $16 \%$ en 1912 . Entre 1870 y 1913, la diferencia de precio entre Londres y Boston de las lanas y los cueros cayó de 59 a $28 \%$ y de 28 a $9 \%$, respectivamente. A estas cifras habría que agregar el impacto de los cambios cualitativos: la incidencia del acortamiento de los viajes sobre la conservación de las mercaderías, la introducción del sistema de refrigerado a bordo, etc.

La productividad del transporte terrestre varía en importancia, obviamente, de acuerdo a las realidades geográficas y extensión de cada región. En regiones como la uruguaya, en la que no hay obstáculos naturales importantes, las ganancias por mejoras en los transportes son notoriamente menos importantes que en países como Colombia, plagado de accidentes geográficos que dificultan las comunicaciones. En el caso uruguayo, un país pequeño y de geografía fácil, el índice de precios del sector transporte (incluye

3 Ver BAPTISTA, B.; BÉRTOLA, L. Uruguay 1870-1913: indicadores de comercio exterior. In: EL DESARROLLO HISTÓRICO DE LAS ECONOMÍAS LATINOAMERICANAS EN PERSPECTIVA COMPARADA, 1999, Montevideo. Anais... Montevideo: Segundas Jornadas de Historia Económica, 1999. 
ferrocarriles, tranvías y ómnibus y no comprende el efecto de sustituir los transportes tradicionales por los modernos) cayó de 228 en 1870 a 100 en 1913; en términos "reales", en este caso comparado con el deflactor implícito del PBI, el precio del transporte de 1913 era el 27\% del de 1870 (BÉRTOLA, 1998 , p. 56 y 58).

La productividad de la producción agropecuaria es de difícil estimación, dada la falta de información acerca de los distintos factores de producción en juego. Estas mejoras tuvieron que ver con la mejora y adaptación de las semillas, la cruza y mestizaje de ganado, el control de plagas y enfermedades, la combinación de la cría de acuerdo al tipo de pasturas, etc. Si tomamos como un indicador de la productividad al peso del ganado vendido para faena, el aumento de la productividad en la ganadería uruguaya habría sido del $86 \%$ entre 1870 y 1913 (BÉRTOLA, 1998, p. 37).

La productividad emergente de los procesos de consolidación institucional es de muy difícil cuantificación, aunque de muy fácil percepción. Probablemente las ganancias de productividad por este concepto estén presentes en las otras que hemos presentado, especialmente en lo que refiere al transporte terrestre y a la producción ganadera: disminución del impacto de las guerras civiles, incremento de la fuerza pública haciendo valer los derechos de propiedad frente al adversario político y el abigeato, disminución de los gastos destinados a la función de vigilancia privada, etc. Supongamos que los hemos integrado en los tres anteriores. ¿Cuál es el aumento agregado de la productividad en 1870-1913? Veamos un simulacro en el cuadro 1.

Cuadro 1 - AUMENTO DE LA PRODUCTIVIDADE DEL SETOR EXPORTADOR, 1870-1913

\% anual ponderación 1870 ponderación 1913 contribución (\%)

$\begin{array}{lcccc}\text { 1) transporte interoceánico } & 0,7 & 8 & 10 & 5 \\ \text { 2) Transporte terrestre } & 3,1 & 21 & 10 & 25 \\ \text { 3) Producción agropecuaria } & 1,1 & 72 & 80 & 70 \\ \text { Total } & 1,3 & 100 & 100 & 100\end{array}$

FUENTES: 1 - Índice del precio real de los fletes, según Isserlis (1938, cuadro VIII, p. 122, col. 2 y 6); 2 - movimiento del índice relativo de precios del transporte terrestre y el deflactor implícito del PBI, Bértola (1998, p. 56 y 58); 3 - peso promedio del ganado para faena, según Bértola (1998, p. 37).

Este crecimiento de la productividad de acuerdo a esta aproximación parece ser importante. El PBI per capita de Uruguay creció a en ese período en torno al $1 \%$ anual, de manera similar al de Gran Bretaña, 
aunque sensiblemente menor al de EUA $(1,81) .{ }^{4}$ La productividad total de los factores fue similar en EUA y Gran Bretaña, lo que está denotando que en EUA la incorporación de capital fue mucho más importante que en Gran Bretaña, ${ }^{5}$ permitiendo el mayor aumento del PBI per capita.

Un rasgo característico del sistema mundial creado en este período fue la complementariedad interindustrial, es decir, la de un comercio internacional basado en el intercambio de materias primas y alimentos por bienes manufacturados. El buen desempeño de la productividad argentina y uruguaya encontraba su contrapartida en una demanda creciente de alimentos y materias primas en Europa, cuya producción se veía crecientemente amenazada por el aumento de la productividad agregada de todo el proceso exportador.

Sin embargo, este proceso no sería eterno, en ninguno de sus componentes. Sabido es que el comercio mundial, que a mediados del Siglo XIX veía crecer las materias primas y alimentos a un ritmo mayor que el de las manufacturas, fue cambiando de estructura a favor de los bienes industrializados. Ello debido al desarrollo industrial, al crecimiento del ingreso, a los cambios en la estructura de la demanda y también a diversas consecuencias del desarrollo tecnológico que reducía la intensidad del uso de materias primas y combustibles (ROWTHORN; WELLS, 1987). A ello debe sumársele la disminución del ritmo de crecimiento de la población europea.

Desde el punto de vista de la oferta también se produjeron cambios importantes. En primer lugar, un gran aumento de la competencia por la incorporación de muy extensas regiones produciendo para el mercado mundial. Sin embargo, si continuamos con nuestro razonamiento de abordar la competitividad de los sectores exportadores latinoamericanos en términos de procesos integrados productivos y comerciales, a partir de la segunda década del Siglo XX ya es posible notar cambios importantes en el movimiento de la productividad. Aún a sabiendas de que las proxies que estamos manejando son sumamente discutibles, la magnitud de los cambios que percibimos nos dejan a salvo del ajuste de las cifras. Como vemos en el cuadro 2 el movimiento de las tres variables que mencionamos nos presenta un panorama muy oscuro para esa modalidad de inserción internacional. El transporte interoceánico enfrenta un proceso de relativo encarecimiento, lo

4 MADDISON, A. Monitoring the world economy, 1820-1992. [S. 1.: s. n., 19-]. Table K. 2.

5 La inversión en capital físico, exceptuando la vivienda, creció al 5,53\% anual en EUA y al 1,73\% en el Reino Unido. MADDISON, op. cit., table K. 2. 
que daña la capacidad de competencia de las regiones alejadas en relación a la de los mercados de destino. El aumento del precio de los fletes fue provocado originalmente por la guerra mundial pero con posterioridad sus niveles se mantuvieron, en términos relativos, muy por encima del del resto de los precios. Esto constituye un cambio radical con respecto al período anterior. Un proceso muy similar encontramos en el plano de los transportes terrestres domésticos. Los precios de este sector se incrementaron un 1.4\% anual por encima del de otros precios de la economía. Finalmente la productividad agropecuaria, aproximada por el peso del ganado, experimentó una caída. Si bien esta caída puede haber obedecido a aspectos circunstanciales, puede sostenerse que las mayores posibilidades de aumento de la productividad por la vía de la incorporación de innovaciones universales como las ya señaladas, se habían visto agotadas, y el aumento de la productividad quedaría librado a las posibilidades del más complejo proceso de ampliación de la capacidad alimenticia de la pradera natural, proceso que demanda de arreglos institucionales y elementos sistémicos que habrían de demostrarse sumamente complejos en el desarrollo agrario durante el resto del siglo.

Igualmente podemos señalar, como una contribución a todos los aspectos señalados, que las ganancias de productividad provenientes de la consolidación política e institucional ya habían dado sus resultados y dejaban de tener un impacto fuerte en el aumento de la productividad.

Cuadro 2 - AUMENTO DE LA PRODUCTIVIDADE DEL SECTOR EXPORTADOR, 1913-1930

\% anual ponderación 1913 ponderación 1930 contribuición (\%)

$\begin{array}{lcccc}\text { 1) Transporte interoceánico } & -0,4 & 10 & 11 & 4 \\ \text { 2) Transporte terrestre } & -1,4 & 10 & 10 & 12 \\ \text { 3) Producción agropecuaria } & -1,2 & 80 & 79 & 84 \\ \text { Total } & -1,1 & 100 & 100 & 100\end{array}$

FUENTES: 1) Indice del precio real de los fletes, según Isserlis (1938, Cuadro VIII, p. 122, col. 2 y 6); 2) movimiento entre el índice relativo de precios del transporte terrestre y el deflactor implícito del PBI, Bértola (1998, 56 y 58); 3) peso promedio del ganado para faena, según Bértola $(1998,37)$. 
Sabido es que las economías exportadoras estuvieron fuertemente expuestas a las fluctuaciones económicas y financieras internacionales. Los desequilibrios externos sometieron el modelo de crecimiento a fuertes fluctuaciones. Las restricciones externas hasta 1913 se vieron atenuadas por el movimiento positivo de los términos de intercambio. Recientemente hemos estimado una mejora de los mismos en más de un $100 \%$ entre 1870 y 1913 (BAPTISTA; BÉRTOLA, 1999). Entre 1913 y 1930 los términos de intercambio de Uruguay cayeron un 20\% (BÉRTOLA, 1991).

Otro factor importante que contribuyó a compensar las tendencias al déficit de la balanza comercial fue la entrada de capitales. Es sabido que en los años '20 este componente fue fundamental para mantener el equilibrio, a la vez que contribuyó a ocultar los profundos desequilibrios presentes en la economía mundial (THORP, 1991). La desaparición de esta fuente de financiación a partir de los años '30 constituyó uno de los elementos que determinaron el muy fuerte impacto de la crisis.

Sin duda nos están faltando trabajos que nos den cuenta en términos comparativos de los niveles de productividad. Es un esfuerzo fundamental el de determinar, por ejemplo, el origen de las diferencias de productividad entre las economías del Sur y la de EUA. Existe una obvia diferencia entre ambos. Más allá de las ventajas que EUA pudiera tener en la producción primaria y las ventajas de localización en relación a las economías del Sur con respecto a los mercados europeos, EUA logró en el período de expansión agroexportadora sentar las bases de su liderazgo en el plano industrial, experimentando en este sector un proceso de muy rápido aumento de la productividad. Más aún, lo que se produce en 1880-1890 es el surgimiento de un nuevo "estilo tecnológico" (PÉREZ, 1983), caracterizado por:

- el sistemático uso de la electricidad como fuerza motriz a costos rápidamente decrecientes;

- la sustitución del hierro por el acero como principal material de construcción de ingeniería mecánica con una oferta muy abundante;

- el desarrollo de ramas de infraestructura como la ingeniería y mecánica eléctrica, la producción de cables, ingeniería pesada y de los armamentos, astilleros en base a acero, industrias químicas pesadas, colorantes sintéticos y producción de electricidad;

- el rápido crecimiento de ramas como la construcción de aviones, automóviles, telecomunicaciones, radio, productos 
de aluminio, bienes de consumo durable, petróleo y plásticos;

- el desarrollo de firmas gigantes, cárteles, trusts, mercados oligopolizados y monopolizados, la concentración de las finanzas y la banca (FREEMAN, 1989).

Dejando a un lado las dificultades institucionales creadas por el colapso del patrón oro y la falta de un sistema internacional de pagos, el nuevo mundo que estaba surgiendo habría de generar bases de competitividad y desarrollo de la productividad completamente diferentes. Habría de generar radicales cambios de la estructura de la producción y el consumo. Las nuevas ramas industriales dinámicas y con fuerte crecimiento de la productividad habían cambiado.

¿Cuán bien preparados estaban los países del Sur para esta nueva etapa? Una respuesta adecuada a esta pregunta demanda un trabajo muy serio. Tomemos solamente como una referencia la comparación de nuestros niveles educativos. A principios de siglo, la cobertura educativa de Argentina y Uruguay era menos de la mitad que la de los países desarrollados. La de Brasil un 20\%. No se precisa hacer un gran esfuerzo para inferir las implicancias que estas diferentes dotaciones de "capital humano" puedan tener en relación al desarrollo posterior.

Por otra parte, la cobertura educativa, además de ser un indicador de los esfuerzos domésticos de aprendizaje, puede ser tomada como un indicador de equidad. Tylecote (1992) ha enfatizado el rol jugado por la distribución del ingreso en la forma en que se difunden los diferentes estilos tecno-económicos a regiones periféricas de la economía mundial. Tylecote y Lingärde (1998) sostienen que la estructura relativamente igualitaria de distribución del ingreso y los correspondientes altos niveles educativos de la población han sido determinantes de la rápida difusión del estilo tecnoeconómico del acero y la electricidad a los países escandinavos, en relación con los del Sur de América. A su vez, esa rápida absorción del nuevo estilo tecnológico habría sido la base de su posterior incorporación al núcleo de países tecnológicamente líderes.

Nuestra información sobre salarios reales internacionales nos indica que, a pesar del rápido crecimiento de la productividad, los salarios reales rioplatenses quedaron siempre a gran distancia de los pagados en EUA y también Canadá. Un estudio en profundidad debería determinar la contribución de diferentes componentes a esa situación: la productividad, por un lado, y las estructuras de los mercados de tierras y trabajo por otro.

No debería ignorarse la abundante bibliografía sobre la modalidad oligárquica de ocupación territorial en América Latina. La reacción contra la 
concentración de la riqueza en la era de la globalización de fines del siglo pasado, que a menudo fue enfrentada con sueños de autarquía, se basó sin embargo en elocuente evidencia sobre formas y estructuras de la propiedad que frenaron el desarrollo de la productividad. Recientes trabajos sobre la temática enfatizan, además, que estos patrones de propiedad territorial, especialmente en Argentina y Brasil, no fueron principalmente una herencia colonial, sino principalmente resultado de relaciones de poder consolidadas en la segunda mitad del Siglo XIX, en estrecha interacción con el orden económico emergente (OSORIO; SECRETO, 1998).

Una comparación entre la pampa argentina y las praderas canadienses concluye que la interacción entre regímenes de relaciones de propiedad y estrategias individuales produce consecuencias de largo plazo imprevisibles para los actores: "by buttressing property relations based on owner-occupation of the means of production, individuals undermined the financial stability of the regional economy of the prairies; and by enhancing the estancia-dominated pampas, individuals inhibited the development of technology or the forces of production." ${ }^{\prime 6}$ Con respecto a los mercados de trabajo, se señala que en Canadá la estrategia predominante fue la del asentamiento de pequeños propietarios en fincas propias. De haber ofrecido y vendido su fuerza de trabajo, esto hubiese demorado el enriquecimiento de los campesinos. De esta manera, la reserva de fuerza de trabajo se vio fuertemente disminuida. Por el contrario, la oferta de fuerza de trabajo en Argentina fue más elástica y abundante. ${ }^{7}$

Thorp recientemente ha resumido el problema en términos precisos: "La primer ola de expansión correspondió sobre todo a un período de crecimiento impulsado por la exportación de productos básicos. Sin embargo, la mano de obra escaseaba, un problema que la inmigración paleó parcialmente. La escasez condujo perversamente no a una buena distribución del ingreso y a un alto rendimiento para el factor trabajo, sino a instituciones que reprimieron y controlaron a los trabajadores y crearon oferta de mano de obra controlando a los campesinos. ${ }^{8}$

6 ADELMAN, J. Frontier development. Land, Labour abd capital in the wheathlands of Argentina and Canada 1890-1914. Clarendon: Clarendon Press-Oxford, 1914. p. 260.

7 Ibid., p. 261

8 THORP, R. Progresso, pobreza y exclusión: Una historia económica de América Latina en el siglo XX. [S. 1.]: BID - Unión Europea, 1998. p. 6. Ver también CARDOSO, C. F.; PÉREZ BRIGNOLI, H. Historia Económica de América Latina, II. Barcelona: [s. n.], 1979; BAUER, A. Rural Spanish America, 1870-1930. In: BETHELL, L. (Ed.). Cambridge history of Latin America IV. [S. 1.: s. n., 19-]. p. 151-186; DUNCAN 
Sabido que el caso uruguayo y argentino fueron parcialmente diferentes, en los que la presencia del trabajo asalariado libre fue mucho más predominante que en otros países latinoamericanos. Sin embargo, al hablar de escasez de mano de obra no debemos dejar de señalar los problemas relativos a la distribución de la propiedad de la tierra, al menos a partir del período de intenso alambramiento de los campos de la segunda mitad del Siglo XIX. En el caso argentino, las fluctuaciones estacionales de un mercado de trabajo fuertemente ligado a la actividad agropecuaria fueron decisivas. Flexibilidad, movilidad espacial y escasa calificación de la mano de obra fueron características sobresalientes del mercado de trabajo argentino (SÁBATO, 1985) y muy probablemente del uruguayo también.

Entonces, la estructura de la propiedad de la tierra, la formación del mercado de trabajo, las características de la formación del Estado nacional (de lo que no hemos hablado), la interacción de la estructura productiva con la dinámica y difusión del cambio tecnológico, las formas de distribución del ingreso y los niveles educativos, son algunos de los elementos explicativos que deben incorporarse a la definción de regímenes de convergencia y divergencia.

\section{A MODO DE CONCLUSIÓN}

Este trabajo, pensado más como una presentación oral que como una ponencia escrita, ha intentado delinear una estrategia de abordaje de la problemática del dispar desempeño económico de las naciones.

Nuestra opción es metodológica y teórica. En el primer caso, aspiramos a interpretar hechos históricos y construir teoría capaz de dar cuenta de ellos. A diferencia de otros intentos que parecen jerarquizar la validación de teorías usando la historia como campo de pruebas, aspiramos a construir teoría historizada e interpretación histórica que haga uso de la teoría de manera sistemática.

Encontramos más fuentes de inspiración en diversos cuerpos teóricos que ponen el proceso de innovación en primer lugar, tanto en el plano de la producción como en el del consumo. Jerarquizamos el rol de los aspectos institucionales en la determinación de las formas de distribución

K.; RUTLEDGE, I. (Ed.). Land and labour in Latin America. Cambridge: [s. n.], 1977; BULMER-THOMAS, V. Latin America economic history since independence. Cambridge: [s. n.], 1994. Chapter 4. 
de los frutos del aumento de la productividad, ya sea entre salarios, rentas y ganancias, como entre naciones.

Hemos presentado escuetamente diferentes escenarios históricos de convergencia y divergencia de Argentina, Brasil y Uruguay con respecto a los países desarrollados entre 1870 y 1990, en base a trabajos escritos junto a otros colegas. En esos escenarios encontramos importantes similitudes en el comportamiento de los salarios internacionales y el PBI per capita.

Posteriormente nos hemos concentrado en ilustrar un posible abordaje del período 1870-1930, principalmente en base a información de Uruguay, pero teniendo a la región como contexto.

En términos generales podemos concluir que el relativamente exitoso desarrollo de las economías rioplatenses tuvo relación con la difusión del estilo tecnológico del vapor y los ferrocarrilles, que permitió potenciar la producción agraria de las zonas periféricas, en un contexto de rápido crecimiento de la demanda y mal desempeño de la productividad agraria en los países europeos. Al buen desempeño se sumó un rápido crecimiento de la productividad de la propia producción agraria rioplatense, debido a la incorporación de innovaciones tecnológicas de tipo universal, que no alteraban sustancialmente la capacidad forrajera. En este período, se produjo un importante aumento de los términos de intercambio, que junto a la expansión del volumen de las exportaciones permitió sostener un importante crecimiento importador que alimentó consumos diversos e industrias locales.

El período se caracterizó por una tendencia a una distribución regresiva del ingreso en términos de la relación salarios-precios de la tierra, pero la masa salarial creció significativamente, por lo que el PBI per capita y los salarios mostraron movimientos relativos similares, a pesar del movimiento opuesto de la distribución del ingreso en los países europeos.

La discusión en torno a las determinantes de las políticas de cambio estructural en los años ' 30 encuentra evidencia de interés en lo sucedido a partir de aproximadamente la Primer Guerra Mundial. Desde entonces (no hemos buscado precisar el punto de inflexión con precisión en este trabajo) todos los indicadores manejados para ejemplificar el buen desempeño anterior muestran un viraje sustancial. El paradigma que sustentara el desarrollo anterior pierde vigor y las potencialidades de inserción dinámica en la economía internacional de los países del Río de la Plata se erosionan. El nuevo estilo tecnológico del acero y la electricidad encuentra una distribución sectorial de la productividad sumamente distinta, que habría de contribuir a cambiar las bases del comercio internacional. El transporte e intercambio de bienes de primarios habría de dejar lugar en el comercio mundial al intercambio intraindustrial, con bases de competitividad muy diferente. 
No hemos estimado el retraso de la difusión del nuevo paradigma a los países del Sur, pero en términos de cobertura educativa, los niveles alcanzados por los países avanzados en 1930 solamente fueron alcanzados por los países del Sur a finales de la década de 1960.

La irrupción del nuevo paradigma trajo aparejados cambios en las pautas de consumo, en la dinámica demográfica y en diversos arreglos institucionales tendientes a generar los llamados Estados de Bienestar, que contribuyeron a determinar una caída de los términos de intercambio de los países del Sur y a profundizar el carácter estructural de las restricciones externas al crecimiento.

En este contexto, y más allá de las modalidades concretas que adoptaron las políticas de cambio estructural (determinadas obviamente por las características sociales e institucionales heredades del desarrollo anterior), la alternativa parecía clara: solo el cambio estructural podía evitar un rezago creciente con respecto a los países líderes. En un contexto de retracción y proteccionismo en el comercio mundial, la reducción del coeficiente de importaciones parecía ineludible. Para ello, el aprendizaje tecnológico era una condición básica. Las condiciones para competir, desde el inicio, eran sumamente difíciles. A la luz de este punto de partida debería evaluarse el desempeño de ese período.

\section{RESUMEN}

A partir de una presentación del desempeño de las economías de Argentina, Brasil y Uruguay en relación a un núcleo de economías desarrolladas en 1870-1990, este trabajo se concentra en explicar el cambio desde una posición de relativamente buen desempeño de las economías rioplatenses hasta principios del Siglo XX, hacia un proceso de progresiva divergencia. El abordaje enfatiza el estudio del crecimiento de la productividad de las economías agro-exportadoras en relación a los cambios tecnológicos e institucionales producidos a lo largo de toda la cadena de producción y comercialización nacional e internacional. Igualmente se refiere a las características de los mercados de factores para la determinación de patrones de distribución del ingreso, que afectan niveles relativos del PBI per capita y de los salarios en términos de paridad de poder de compra. Las determinantes de un buen desempeño hasta la década de 1910 parecen revertirse entre 1910-1930, de la mano de cambios profundos en los paradigmas tecno-económicos y de fuertes cambios socio-institucionales en las economías desarrolladas. Parece agotarse una modalidad de inserción 
internacional basada en el uso intensivo de factores naturales combinados con la revolución de los transportes terrestres y marítimos, a la vez que la herencia en términos de formación de capital humano dejada por el modelo agro-exportador augura muy fuertes dificultades para insertarse en los nuevos patrones de competencia internacional. Palabras-clave: divergencia, productividad, distribución del ingreso, cambio tecnológico, Argentina, Brasil, Uruguay.

\begin{abstract}
On the basis of an overview of Argentine, Brazilian and Uruguayan growth in 1870-1990 and in relation to a core of developed countries, this paper focuses on the transition which Argentina and Uruguay went through, from a high relative performance to a process of cumulative divergence. The approach emphasizes the role of productivity growth in the agrarian export sector, as related to technological and institutional changes along the whole national and international productive and commerce chain. The paper also refers to the features of factor markets in shaping the pattern of income distribution, and on real per capita GDP and purchasing power parity real wage levels. The underlying forces of the relatively successful performance until the 1910s seem to have experienced a reversal in 1910-1930, together with deep changes in the techno-economic paradigm as well as in the socio-institutional environment in the developed economies. The pattern of foreign trade, based on an intensive use of natural resources combined with the benefits of the transport revolution, both terrestrial and maritime, seemed to have come to $\mathrm{n}$ end. The heritage of the agrarian export-led growth in terms of poor human capital formation, anticipates the difficulties which those economies should face in relation to the emerging patterns of international competition.

Key-words: divergence, productivity, income distribution, technical change, Argentina, Brazil, Uruguay.
\end{abstract}




\section{REFERENCIAS}

ABRAMOVITZ, M. Catching Up, Forging Ahead and Falling Behind. [S. 1.: s. n., 19-].

ADELMAN, J. Frontier Development. Land, labour and capital in the Wheatlands of Argentina and Canada 1890-1914, Oxford Historical Monographs. Oxford: Clarendon Press, 1994.

BAUER, A. Rural Spanish America, 1870-1930. In: BETHELL, L. (Ed). Cambridge History of Latin America IV. [S. 1.: s. n., 19-]. p. 151-186.

BAPTISTA, B.; BÉRTOLA, L. Uruguay 1870-1913: Indicadores de Comercio Exterior. In: EL DESARROLLO HISTÓRICO DE LAS ECONOMÍAS LATINOAMERICANAS EN PERSPECTIVA COMPARADA, 1999. Anales... [S. 1.]: Segundas Jornadas de Historia Económica, julio de 1999.

BÉRTOLA, L. El PBI uruguayo 1870-1936 y otras estimaciones. Montevideo: Facultad de Ciencias Sociales, 1998.

BÉRTOLA, L. La Industria Manufacturera Uruguaya 1913-1961. Un enfoque sectorial de su crecimiento, fluctuaciones y crisis. Montevideo: [s. n.], 1991.

BÉRTOLA, L.; BERTONI, R. Educación y aprendizaje: su contribución a la definición de escenarios de convergencia y divergencia. In: EL DESARROLLO HISTÓRICO DE LAS ECONOMÍAS LATINOAMERICANAS EN PERSPECTIVA COMPARADA, 1999. Anales... [S. 1.]: Segundas Jornadas de Historia Económica, julio de 1999.

BÉRTOLA, L.; CAMOU, M.; PORCILE, G. Comparación Internacional del Poder Adquisitivo de los Salarios Reales de los Países del Cono Sur, 1870-1945. In: MERCADO DE TRABAJO Y NIVEL DE VIDA, 1999. Anales... [S. 1.]: Segundas Jornadas de Historia Económica, julio de 1999.

BÉRTOLA, L.; PORCILE, G. Argentina, Brazil, Uruguay and the World Economy: an approach to different convergence and divergence regimes. Montevideo: DT 42, FCS, 1998.

BÉRTOLA, L., et al. Southern cone real wages compared: a purchasing power parity approach to convergence and divergence trends, 1870-1996. [S. 1.]: DT 43, Unidad Multidisciplinaria, Facultad de Ciencias Sociales, Febrero de 1998.

BÉRTOLA, L.; BITTECOURT, M.; PORCILE, G. A Lei de Thirlwall: aplicacaçäo de um modelo VAR a economia brasileira do Pós-Guerra. In: III CONGRESSO BRASILERIO DE HISTÓRIA ECONÔMICA, Curitiba: [s. n.], 1999.

BULMER-THOMAS, V. Latin American Economic History since Independence. Cambridge: [s. n.], 1994.

CARDOSO, C. F. S.; PÉREZ BRIGNOLI, H. Historia Económica de América Latina, II. Barcelona: [s. n.], 1979.

CIMOLI, M. Technological Gaps and Institutional Asymmetries in a North-South Model With a Continuum of Goods. [S. 1.: s. n., 19-].

CRAFTS, N. F. R. Quantitative Economic History, (Working Papers in Economic History 48/99). London: London School of Economics and Political Science, Jan. 1999. 
DE LA FUENTE. Notas sobre la Economía del Crecimiento, I y II. Barcelona: Universidad Autónoma de Barcelona, 1996.

DUNCAN, K.; RUTLEDGE, I. (Ed). Land and Labour in Latin America. Cambridge: [s. n.], 1977.

FREEMAN, CH. The third Kondratieff wave: age of steel, electrification and Imperialism. In: BOHLIN, J. et. al. Samhällsvetenskap, ekonomi och historia. Festskrift till Lars Herlitz. Göteborg: [s. n.], 1989. p. 281-318.

HATTON, T.; WILLIAMSON, J. Latecomers to mass emigration: the Latin experience. In: HATTON, T.; WILLIAMSON (Ed). Migration and the International Labor Market 1850-1939. Routledge: [s. n.], 1994. p. 55-71.

ISSERLIS, L. Tramp Shipping Cargoes and Freights. Journal of the Royal Statistical Society, CI, 1938.

LINGÄRDE, S.; TYLECOTE, A. Resource rich countries in a comparative perspective: nordic countries versus Argentina, Uruguay and Brazil. In: INTERNATIONAL ECONOMIC HISTORY CONGRES, 12., 1998, Madrid. Anais... Madrid: [s. n.], Aug. 1998.

LUNDVALL, B. A. (Ed.). National systems of innovation - toward a theory of innovation and innovative learning. [s. 1.]: Pinter Publishers, 1992.

MADDISON, A. Monitoring the world economy, 1820-1992. [S. 1.: s. n., 19-].

MCCOMBIE, J. S. L.; THIRLWALL, A. P. Economic growth and the balance of payments constraint. [S. 1.: s. n., 19-].

NELSON, R. R. National Systems of Innovation. A Comparative Study. Oxford: [s. n.], 1992.

NELSON, R. Economic growth via the coevolution of technology and institutions. In: LEYDESDORFF, L.; VAN DEN BESSELAAR, P. (Eds.). Evolutionary economics and chaos theory: new directions in technology studies. London: Pinter, 1994.

NORTH, D. The new institutional economics and development (ewp-eh/9309002).

OSORIO SILVA, L.; SECRETO, M. V. Elements for a Comparative History of Private Occupation of Public Land in Argentina and Brazil. Campinas: Unicamp, 1998.

PASINETTI, L. L. Structural Change and Economic Growth: a Theoretical Essay on the Dynamics of the Wealth of Nations. Cambridge: [s. n.], 1983.

PERES COSTA, W. Primary export economy and patterns of state building in Argentina and Brazil. In: INTERNATIONAL ECONOMIC HISTORY CONGRESS, 12., Paper...[S. 1.: s. n., 19-]. (Session: 1870-1990: product and income growth rates convergence and divergence within originally land-abundant Latin American countries and between them and West-European countries).

PEREZ, C. Structural Change and the Assimilation of New Technologies in the Economic and Social System. Futures, n. 4, p. 357-375, 1983.

REINERT, E. S., Competitiveness and its Predecessors - a 500-year Cross-National Perspective, Structural Change and Economic Dynamics, n. 6, p. 23-42, 1996.

ROWTHORN, R. E.; WELLS, J. De-Industrialization and Foreign Trade. CambridgeNueva York-Melbourne: [s. n.], 1987. 
SÁBATO, H. La formación del mercado de trabajo en Buenos Aires, 1850-1880. Desarrollo Económico, v. 24, n. 96, p. 561-592, 1985.

THIRLWALL, A. P. Foreign Trade Elasticities in Centre-Periphery Models of Growth and Development. Quarterly Review Banca Nazionale del Lavoro, n. 146, 1983.

THORP, R. América latina y la economía mundial desde la primera guerra mundial hasta la depresión mundial. In: BETHELL, L. (Ed.). Historia de América Latina. 7. América latina: economía y sociedad, c. 1870-1930. [S. 1.]: Crítica, 1991.

THORP, R. Progreso, pobreza y exclusión: una historia económica de América Latina en el Siglo XX. [S. 1.]: BID-Unión Europea, 1998 (English version, Progress, poverty and exclusion: an Economic History of Latin America in the $20^{\text {th }}$ Century. [S. 1.]: InterAmerican Development Bank, 1998).

TYLECOTE, A. The Long Wave in the World Economy. [S. 1.: s. n.], 1992.

VERSPAGEN, B. Uneven Growth Between Interdependent Economies. [S. 1.: s. n., 19-].

WILLIAMSON, J. Real Wages and Relative Factor Prices in the Third World 18201940: Asia. Harvard Institute of Economic Research, Discussion Paper Number 1844, July 1998.

WILLIAMSON, J. Real Wages and Relative Factor Prices in the Third World 18201940: Latin America. Harvard Institute of Economic Research, Discussion Paper Number 1853, November 1998.

WILLIAMSON, J. Real Wages and Relative Factor Prices in the Third World 18201940: The Mediterranean Basin. Harvard Institute of Economic Research, Discussion Paper Number 1842, July 1998.

WILLIAMSON, J. Growth, Distribution and Demography: Some Lessons from History. Explorations in Economic History, n. 3, p. 241-271, 1998.

WILLIAMSON, J. The Evolution of Global Labor Markets Since 1830: Background Evidence and Hypothesis. Explorations in Economic History, n. 3, 1995. 